\title{
Article
}

\section{Validation of an Automated Screening System for Diabetic Retinopathy Operating under Real Clinical Conditions}

\author{
Soledad Jimenez-Carmona ${ }^{1, *(\mathbb{D})}$, Pedro Alemany-Marquez ${ }^{1, *(\mathbb{D}}$, Pablo Alvarez-Ramos ${ }^{1}$, Eduardo Mayoral ${ }^{2}$ \\ and Manuel Aguilar-Diosdado ${ }^{3}$ \\ 1 Ophthalmology Department, Hospital Universitario Puerta del Mar, University of Cadiz, \\ 11009 Cadiz, Spain; pablo.alvarez.ramos@gmail.com \\ 2 Comprehensive Healthcare Plan for Diabetes, Regional Ministry of Health and Families of Andalusia, \\ Government of Andalusia, 41020 Seville, Spain; eduardo.mayoral.sspa@juntadeandalucia.es \\ 3 Biomedical Research and Innovation Institute of Cadiz (INiBICA), 11009 Cadiz, Spain; \\ manuel.aguilar.sspa@juntadeandalucia.es \\ * Correspondence: Soledad.jimenez@uca.es (S.J.-C.); pedromaria.alemany@uca.es (P.A.-M.)
}

check for

updates

Citation: Jimenez-Carmona, S.;

Alemany-Marquez, P.;

Alvarez-Ramos, P.; Mayoral, E.;

Aguilar-Diosdado, M. Validation of

an Automated Screening System for

Diabetic Retinopathy Operating

under Real Clinical Conditions. J.

Clin. Med. 2022, 11, 14. https://

doi.org/10.3390/jcm11010014

Academic Editor: Kei Nakajima

Received: 22 November 2021

Accepted: 16 December 2021

Published: 21 December 2021

Publisher's Note: MDPI stays neutral with regard to jurisdictional claims in published maps and institutional affiliations.

Copyright: (C) 2021 by the authors. Licensee MDPI, Basel, Switzerland. This article is an open access article distributed under the terms and conditions of the Creative Commons Attribution (CC BY) license (https:// creativecommons.org/licenses/by/ $4.0 /)$.

\begin{abstract}
Background. Retinopathy is the most common microvascular complication of diabetes mellitus. It is the leading cause of blindness among working-aged people in developed countries. The use of telemedicine in the screening system has enabled the application of large-scale population-based programs for early retinopathy detection in diabetic patients. However, the need to support ophthalmologists with other trained personnel remains a barrier to broadening its implementation. Methods. Automatic diagnosis of diabetic retinopathy was carried out through the analysis of retinal photographs using the 2iRetinex software. We compared the categorical diagnoses of absence/presence of retinopathy issued by family physicians (PCP) with the same categories provided by the algorithm (ALG). The agreed diagnosis of three specialist ophthalmologists is used as the reference standard $(\mathrm{OPH})$. Results. There were 653 of 3520 patients diagnosed with diabetic retinopathy (DR). Diabetic retinopathy threatening to vision (STDR) was found in 82 patients (2.3\%). Diagnostic sensitivity for STDR was $94 \%$ (ALG) and 95\% (PCP). No patient with proliferating or severe DR was misdiagnosed in both strategies. The k-value of the agreement between the ALG and OPH was 0.5462, while between PCP and OPH was 0.5251 ( $p=0.4291)$. Conclusions. The diagnostic capacity of 2iRetinex operating under normal clinical conditions is comparable to screening physicians.
\end{abstract}

Keywords: diabetic retinopathy; teleophthalmology; diagnostic accuracy; population-based screening; sight-threatening diabetic retinopathy

\section{Introduction}

In 2019, the IDF (International Diabetes Federation) estimated that 463 million adults worldwide suffered from diabetes, and projected the number to rise to 700 million by 2045 [1]. The prevalence of diabetes in Andalusia, the most populated autonomous community in the south of Spain, is higher (15.3\%) than in the rest of Spain $(12.5 \%)$, in close relation to lifestyle and socioeconomic factors [2].

Retinopathy is the most common microvascular complication in patients with diabetes mellitus [3]. In developed countries, diabetic retinopathy (DR) is one of the leading causes of blindness among people of working age [4]. A recent meta-analysis has calculated that in diabetic patients aged 20-79 years, the overall prevalence of any DR is 35\% [5]. While the global prevalence of DR and Diabetic Macular Edema (DME; potential complication of DR), for the period 2015 to 2019 were 27.0\%, and this prevalence in Europe was estimated to be $20.6 \%$, calculated from the results of population-based studies with retinography [6]. It has been known for decades that proper treatment of DR decreases the incidence of severe visual loss when early diagnosed [7]. Telemedicine systems enable the remote analysis of digital fundus photographs, thus detecting the presence of DR lesions. Based on this 
technology, population-based screening programs have been developed in different countries [8]. The growing number of diabetic patients and their periodic medical monitoring entails an increase of these DR-detection digital analyses. Due to the limited number of ophthalmologists, other professionals are required to address DR screening. In particular, these range from family physicians, endocrinologists or nurses in high-income countries to trained non-medical personnel in middle-income countries [9-13].

In recent years, the research effort has focused on the development of automated diagnostic strategies that can complement or replace screening personnel, which would help reduce the workload and improve access for diabetic patients requiring early diagnosis $[14,15]$.

The Andalusian Public Health System (APHS) provides universal health care to the 8.4 million inhabitants of Andalusia, which represent $18 \%$ of the Spanish population. The APHS encompasses an extensive network, with two levels of care (1500 primary healthcare centers and 49 hospitals) based on accessible, high-quality, patient-centered care, in a system with universal coverage and funded by taxation. The APHS, through the program for Early Detection of Diabetic Retinopathy (APDR), which is part of the Comprehensive Healthcare Plan for Diabetes (CHPD), provides a network of digital desktop fundus cameras placed in primary healthcare centers throughout the region. The screening system developed by APHS consists of two phases: in the first phase, digital fundus photographs of diabetic subjects with no previous diagnosis of DR are recorded with a non-mydriatic retinal camera (NMRC). Secondly, the primary care physicians (PCP) of each center assess these photographs, and then, both those displaying a probable DR diagnosis and inconclusive ones are sent via a specific intranet to a reference ophthalmologist for diagnostic confirmation. From January 2005 to June 2019, 888,318 examinations were performed, corresponding to 429,791 patients [2]. It should be noted that patients with a healthy retina are examined periodically, which increases the number of images under study every year.

To reduce this increasing workload of PCP performing the screening activity, the Lynch Diagnostics (Granada, Spain) (LD) computer-aided diagnostic platform, in collaboration with APHS, developed the 2iRetinex algorithm. The 2iRetinex software (Granada, Spain) lassifies the screening images according to their quality. Specifically, for the non-rejected images, it provides information on the number, type and location of the lesions and, ultimately, a final diagnostic decision.

The aim of this research study is the validation of the 2iRetinex software as a complement or substitute for the screening physician. The clinical diagnosis of the screening physician is formulated in terms of the absence or presence of DR. To perform the comparison of this categorical diagnostic strategy with the same categories provided by the algorithm, we introduced the algorithm into the APDR system. The algorithm extracted the diagnosis of the presence or absence of diabetic retinopathy from the system, obviating the additional information. The agreed diagnosis of three specialist ophthalmologists is used as the reference standard ("ground truth") to measure its clinical performance.

\section{Materials and Methods}

\subsection{Study Participants from APDR}

An analytical study of diagnostic tests was performed on the fundus images of diabetic patients regularly attending the Andalusian program for early diagnosis of diabetic retinopathy (APDR) circuit. The used protocols were approved by the Ethical Committee for Research and Clinical Trial of Hospital Puerta del Mar (Cadiz, Spain), number 62/16.

To compare the diagnostic capability of the 2iRetinex algorithm (ALG) with that of the screening PCP under usual clinical conditions, we calculated the required sample based on a previous APDR study with a prevalence of retinopathy in diabetic patients of $30 \%$ [16]. To obtain an average sensitivity of $85 \%$ and specificity of $82 \%$ for the screening PCP [16] and a sensitivity of $90 \%$ and a specificity of $93 \%$ for the ALG (internal unpublished data from the company, on a random sample of 575 patients in 2015), as well as, a type 1 and 2 error at 0.5 and at 0.2 , respectively, the sample size estimated was 2421 patients. For a paired 
sample, the required sample size calculation was 2297 patients. Furthermore, as the rate of ungradable (UNG) images was estimated to be $10 \%$, an additional 242 and 230 patients were added, for independent and paired samples, respectively [16].

Retinographies were obtained from all diabetic patients attending ten primary care health centers in the region of Andalusia (Spain) included in the APDR patient flow circuit using a standardized protocol previously described [16-18]. Briefly, three photographs of each eye were recorded using a retinal camera. The following camera models were used: the Topcon NW 200 (Topcon, Tokyo, Japan) in seven of the ten study centers and in the remaining three centers, the Topcon NW100 (Topcon, Tokyo, Japan), the Zeiss VISUCAM (Carl Zeiss Meditec AG, Jena, Germany) and DRS retinography, respectively. Then, the PCP analyzed the images stored on the APDR server. The images of the patients classified as UNG and those that present findings of DR are assigned, through the intranet, to the reference ophthalmologist who issued a clinical judgment of confirmation. If no DR lesions were detected (NODR) the patient continued in the screening system for future examinations.

\subsection{2iRetinex Software}

The 2iRetinex software (patent number RPI201499900601833) extracted different features from the retinographies. Specifically, 2iRetinex extracted the vascular tree through the isolation of the green channel and microaneurysms and hemorrhages (which are candidates for lesions by applying structural characteristics), through analysis of the dark residual objects. In addition, the 2iRetinex software superimposed a binary mask on the green layer of the images, which made it possible to locate the optic disc. Using a filter also highlighted the bright lesions (exudates and cotton spots). Furthermore, it was able to detect the position of the macula. Finally, a topographic reference system was established to locate the lesions by coordinates. After checking that the image was suitable for analysis, the original image (Figure 1a) was transformed into the resulting image shown in Figure $1 \mathrm{~b}$.

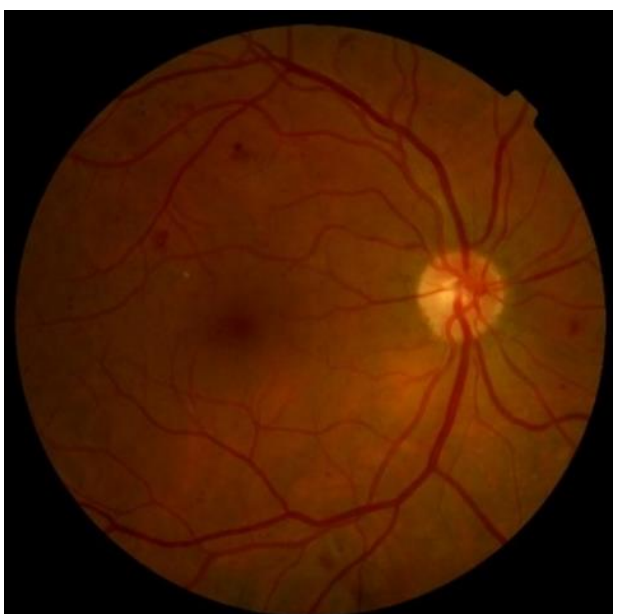

(a)

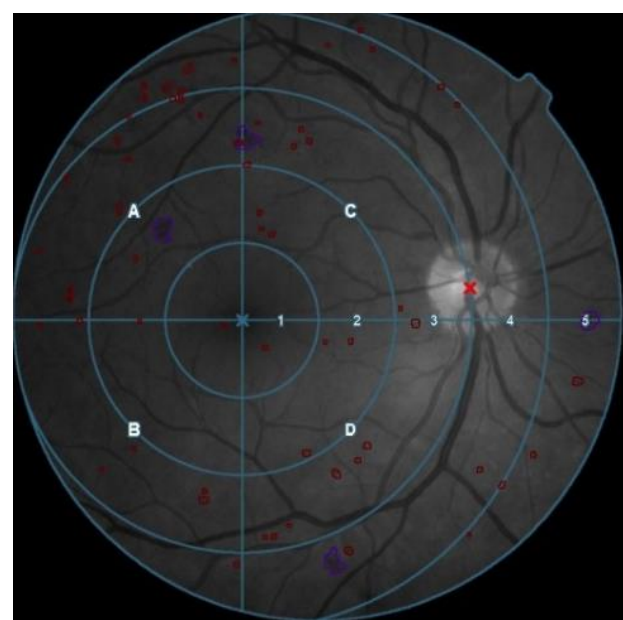

(b)

Figure 1. Software analysis 2iRetinex: (a) A original APDR image of fundus photography of a patient with diabetic retinopathy; (b) Location and type of lesions. Superimposed the coordinates system. Red lesions in red, white lesions in blue.

\subsection{Source of Images and Data}

In the participating centers of this study, between April 2017 and June 2018, a capture system was installed between the retinography and the APDR network. This system allowed the original images in TIFF format to be sent to the LD diagnostic platform to obtain the patient's clinical diagnosis through the 2iRetinex software (ALG) using the same diagnostic categories as the PCP. These images, but not the diagnoses, were available on a server for the ophthalmic researchers (SJC, PAM, PAD). The latter were the ones who established the reference diagnosis (OPH). Simultaneously, the images were returned to 
the APDR network for compression into JPG for final screening by the PCP, following the usual circuit. The PCPs were not aware of the parallel diagnostic systems. Moreover, at no time were the real graders aware of the diagnosis issued by the other study participants before issuing their clinical judgment.

To ensure that the study was double-blind, the LD platform provided an internal control code. Once the OPH and ALG diagnoses were established, the list of codes related to the original health identification was sent to the administrative management of the APDR. Likewise, the demographic data of the study sample and the PCP diagnoses were sent from the APDR for cross-checking.

For each patient, data were obtained for the categorical variables (gender and type of diabetes) and the quantitative variables (age and years of duration of the disease at the time the retinographies were taken; years from diagnosis). The database also recorded the use of pre-exploration mydriatics.

\subsection{Diagnostic Criteria and Convention}

For each patient, the PCP, ALG and OPH diagnosis were obtained, with the same diagnostic categories. The following criteria were used to classify the images:

If the image lacked sufficient quality to confirm or rule out diabetic lesions, it was classified as Ungradable (UNG).

If the image quality was sufficient and no diabetic retinopathy lesions were found, it was classified as No Diabetic Retinopathy (NODR)

Regardless of the quality of the image, if retinopathy lesions were observed, the image was classified as DR.

For the final diagnosis of each patient, as carried out in the APDR circuit, the following criteria were followed:

If at least one of the patient's two eyes was classified as UNG, the patient was diagnosed as UNG.

If DR lesions were detected in the images of at least one eye, the patient was diagnosed as DR.

If none of the images of the patients considered evaluable showed lesions of DR, the patient was diagnosed as NODR.

Research ophthalmologists (SJC, PAM, PAD) classified the stage of DR according to the International Clinical Diabetic Retinopathy Severity Scale [18]. This scale consists of five stages: no diabetic retinopathy (NODR), mild (MILD), moderate (MOD), and severe non-proliferative DR (SEV), and proliferative retinopathy (PROL). The diagnosis of diabetic macular edema (DME) was established by detecting the presence of any signs suggestive or evident of macular edema in at least one eye. Cases diagnosed as DME, SEV and PROL were considered as patients with sight-threatening DR lesions (STDR). If the patient had DR lesions in both eyes, the stage of diagnosis corresponded to that of the eye with the highest degree of DR.

\subsection{Statistical Analyses}

The study data were analyzed using descriptive statistics. Means and standard deviations were calculated for quantitative variables and proportions for qualitive ones. We used the Chi-squared test and the t-test to compare proportions and means, respectively. Sensitivity, specificity, predictive values, and likelihood ratios were used to assess the accuracy of diagnostic tests. Likewise, reliability and agreement were quantified using kappa coefficient. The kappa concordance values of each test (ALG and PCP) were established with respect to the OPH diagnosis and the similarity of the results were examined. The area of the simple ROC curve was calculated for each examiner with respect to ground truth and compared between them. Level of significance was estimated at $p<0.05$.

Statistical analyzes performed using IBM SPSS Statistic v 24 software (Armonk, NY, USA). The sample calculation and the capacity of the diagnostic tests have been quantified using the Epidat 3.1 program (Xunta de Galicia, Galicia, Spain) (www.sergas.es/Saude-publica/EPIDAT). 


\section{Results}

\subsection{Analysis of the Original Sample. Gradable/Ungradable Concordance}

During the download period, image folders of 3575 diabetic patients were obtained from the 10 primary healthcare centers. Due to duplication of files and empty downloads without files, 55 patients were removed. Therefore, the original sample consisted of 3520 image folders, of which $43,9 \%$ corresponded to women (Table 1 ). The average age of the patients was $64.4 \pm 14.6$ years and the mean of the years since the diagnosis of diabetes was $10.37 \pm 7.5$ years. The majority of patients (88.2\%) had type 2 diabetes mellitus (DM2), while patients with type 1 diabetes mellitus (DM1) accounted for $11.4 \%$. In contrast, a minimum proportion $(0.4 \%)$ of the sample corresponded to patients either diagnosed with other categories or without detailed diagnosis (Others).

Table 1. Main characteristics of the study sample obtained from de APDR between April 2017 and June 2018. ${ }^{*}$ Mean SD. DR = diabetic retinopathy. DME = diabetic macular edema. NDME = no diabetic macular edema. STDR = sight-threatening DR.

\begin{tabular}{|c|c|c|c|c|}
\hline \multicolumn{3}{|c|}{ Variable of Interest } & \multicolumn{2}{|c|}{ Study Sample $(n=3520)$} \\
\hline \multicolumn{3}{|c|}{ Gender (F/M) } & \multicolumn{2}{|c|}{$43.9 \% / 56.1 \%(1545 / 1975)$} \\
\hline \multicolumn{3}{|c|}{ Age (years) } & \multicolumn{2}{|c|}{$64.4 \pm 14.6^{*}$} \\
\hline \multicolumn{3}{|c|}{ Years from diagnosis } & \multicolumn{2}{|c|}{$10.4 \pm 7.5^{*}$} \\
\hline \multicolumn{3}{|c|}{ Mydriasis (before photograph) } & $97.8 \%$ & (3443) \\
\hline \multicolumn{3}{|c|}{ Type 2} & $88.2 \%$ & (3103) \\
\hline \multirow[t]{2}{*}{ Diabetes type } & \multicolumn{2}{|c|}{ Type 1} & $11.4 \%$ & $(402)$ \\
\hline & \multicolumn{2}{|c|}{ Others } & $0.4 \%$ & (15) \\
\hline \multicolumn{3}{|c|}{ Ungradable images (UNG) } & $11.6 \%$ & $(407)$ \\
\hline \multicolumn{3}{|c|}{ No diabetic Retinopathy (NODR) } & $69.9 \%$ & $(2460)$ \\
\hline \multirow{7}{*}{$\begin{array}{c}\text { Diabetic } \\
\text { retinopathy (DR) }\end{array}$} & \multicolumn{2}{|c|}{ Mild } & $8.1 \%$ & $(286)$ \\
\hline & \multirow{2}{*}{ Moderate } & NDME & $8.1 \%$ & $(285)$ \\
\hline & & DME & $1.8 \%$ & $(65)$ \\
\hline & \multirow{2}{*}{ Severe } & NDME & $0.1 \%$ & (2) \\
\hline & & DME & $0.2 \%$ & (8) \\
\hline & \multirow{2}{*}{ Proliferative } & NDME & $0.1 \%$ & (3) \\
\hline & & DME & $0.1 \%$ & (4) \\
\hline \multicolumn{3}{|c|}{ STDR } & $2.3 \%$ & $(82)$ \\
\hline
\end{tabular}

Six hundred and fifty-three patients (18,5\%) had images with features of DR. Macular edema was detected in 77 patients $(2.2 \%)$ and DR stage became STDR in 82 patients $(2.3 \%)$. However, it was not possible to establish diagnostic criteria on the condition of the retina in $11.6 \%$ of the images (UNG).

Regarding the distribution by gender, the mean age and mean duration of diabetes were significantly higher among the diabetic females. In addition, in female patients, the proportion of DM1 was higher, and the proportion of DM2 was lower than in the male group. However, there was no significant difference in the prevalence of each stage of DR between genders (Table 2). 
Table 2. Comparison of the study variables between female and male patients. $\mathrm{DR}=$ diabetic retinopathy. DME = macular edema diabetic. STDR = sight-threatening DR. * Results with statistically significant differences.

\begin{tabular}{|c|c|c|c|c|}
\hline \multicolumn{2}{|c|}{ Variable of Interest } & $\begin{array}{c}\text { Female }(n= \\
1545)\end{array}$ & Male $(n=1975)$ & $p$-Value \\
\hline \multicolumn{2}{|c|}{ Age (years) } & $65.4 \pm 15.6$ & $64 \pm 13.8$ & 0.0419 * \\
\hline \multicolumn{2}{|c|}{ Years from diagnosis } & $11.1 \pm 8.1$ & $9.8 \pm 7$ & $<0.0001$ * \\
\hline \multicolumn{2}{|c|}{ Mydriasis } & $97.6 \%$ & $98 \%$ & 0.4196 \\
\hline \multirow{3}{*}{ Diabetes type } & Type 2 & $85.8 \%$ & $90 \%$ & $=0.0001$ * \\
\hline & Type 1 & $13.7 \%$ & $9.6 \%$ & $=0.0001 *$ \\
\hline & Others & $0.5 \%$ & $0.4 \%$ & 0.6579 \\
\hline \multirow{4}{*}{ DR stage } & Mild & $8.1 \%$ & $8.2 \%$ & 0.9143 \\
\hline & Moderate & $10 \%$ & $9.9 \%$ & 0.9216 \\
\hline & Severe & $0.2 \%$ & $0.4 \%$ & 0.2913 \\
\hline & Proliferative & $0.2 \%$ & $0.2 \%$ & 1 \\
\hline \multicolumn{2}{|c|}{ DME } & $2.1 \%$ & $2.2 \%$ & 0.8394 \\
\hline \multicolumn{2}{|c|}{ STDR } & $2.2 \%$ & $2.4 \%$ & 0.6952 \\
\hline
\end{tabular}

Considering the gradable/non-gradable (GRAD/UNG) category of images (previously described in methods), we found that the group of patients with UNG images showed significant differences in the mean age and proportions of the types of diabetes compared to patients with GRAD images (Table 3). Specifically, our data showed that patients with UNG images were 9.3 years older than patients with GRAD images (72. 6 vs. 63. 4). We also found a statistically significantly higher proportion of type 2 diabetic patients and a lower proportion of type 1 diabetic patients, in the group of patients with UNG images (Table 3).

Table 3. Comparison of the common variables between patients with gradable and ungradable images. * Results with statistically significant differences.

\begin{tabular}{|c|c|c|c|c|}
\hline \multicolumn{2}{|c|}{ Variable of Interest } & Gradable & Ungradable & $p$-Value \\
\hline \multicolumn{2}{|c|}{ Gender (F/M) } & $43.8 \% / 56.2 \%$ & $44.2 \% / 55.8 \%$ & 0.8785 \\
\hline \multicolumn{2}{|c|}{ Age (years) } & $63.4 \pm 14.6$ & $72.6 \pm 11.8$ & $<0.0001$ * \\
\hline \multicolumn{2}{|c|}{ Years from diagnosis } & $10.3 \pm 7.3$ & $10.9 \pm 9.2$ & 0.1450 \\
\hline \multicolumn{2}{|c|}{ Mydriasis } & $98 \%$ & $96.6 \%$ & 0.0678 \\
\hline \multirow{3}{*}{ Diabetes type } & Type 2 & $87.2 \%$ & $95.6 \%$ & $<0.0001 *$ \\
\hline & Type 1 & $12.4 \%$ & $4.2 \%$ & $<0.0001 *$ \\
\hline & Others & $0.4 \%$ & $0 \%$ & 0.2012 \\
\hline
\end{tabular}

Regarding the graders used, the images of 407 patients (11.6\%) were classified as UNG according to the consensus diagnosis of the OPHs (Tables 4 and 5). Likewise, the automatic analysis software considered the images of 927 patients $(26.3 \%)$ as inadequate (Table 4), while the PCPs could not provide any diagnosis in 461 patients (13.1\%; Table 5). A significant difference was detected in the proportions of UNG images between ALG and OPH classifier strategies and between ALG and PCP classifier strategies $(p<0.0001)$. The difference of $1.54 \%$ in the proportions of UNG images between OPH and PCP also reached the limit of significance $(p=0.0494)$. 
Table 4. Number of patients in every pair of diagnostic categories comparing ALG vs. OPH.

\begin{tabular}{ccccc}
\hline & OPH-DR & OPH-NODR & OPH-UNG & Total \\
\hline ALG-DR & 455 & 379 & 33 & 867 \\
ALG-NODR & 80 & 1582 & 64 & 1726 \\
ALG-UNG & 118 & 499 & 310 & 927 \\
Total & 653 & 2460 & 407 & 3520 \\
\hline
\end{tabular}

Table 5. Number of patients in every pair of diagnostic categories comparing PCP vs. OPH.

\begin{tabular}{ccccc}
\hline & OPH-DR & OPH-NODR & OPH-UNG & Total \\
\hline PCP-DR & 373 & 233 & 25 & 631 \\
PCP-NODR & 214 & 2005 & 209 & 2428 \\
PCP-UNG & 66 & 222 & 173 & 461 \\
Total & 653 & 2460 & 407 & 3520 \\
\hline
\end{tabular}

The unweighted Kappa statistic was used to assess inter-rater reliability in classifying images into UNG or GRAD. The Kappa statistic shows the observed level of agreement adjusted for the level of agreement that could have occurred by simple chance. A value of $0.75-1.00$ indicates an excellent agreement, $0.4-0.75$ represents a moderate agreement, and lower values display the deficient agreement. We found that the kappa value was 0.3623 for the agreement between ALG and OPH classifier strategies. The agreement between PCP and OPH obtained a k-value of 0.3144 . The kappa homogeneity test showed a Chi-square value of 2.7274 corresponding to a $p=0.0986$. Therefore, the difference in agreement between the two diagnostic strategies was not statistically significant.

Description of the distribution of the diagnostic categories UNG, DR and NODR of the two diagnostic strategies compared to the criteria of the ophthalmologists:

The proportions of the GRAD/GRAD and UNG/UNG agreement categories for ALG versus $\mathrm{OPH}$ were 0.78 and 0.30 , respectively, with a composite agreement ratio of 0.80 . The proportions of the GRAD/GRAD and UNG/UNG agreement categories for PCP versus $\mathrm{OPH}$ were 0.84 and 0.25 , respectively, with a composite ratio of 0.85 .

Overall, these results demonstrated the proportion of UNG was higher in ALG, and the proportion of composite agreement was higher in PCP. It should be noted that the concordance between ALG and OPH was slightly better ( 0.3623 vs. 0.3144$)$ because there was a greater coincidence when classifying UNG images (ALG 0.30 vs. PCP 0.25).

\subsection{Comparison of DR/NODR Diagnostic Category in Unpaired Samples. Diagnostic Validity}

The 407 patients considered as UNG by the OPHs were removed from the original sample. This new sample of 3113 patients was partially described when the comparison between GRAD vs. UNG samples was carried out (Table 3). Based on the results of Tables 4 and 5, two samples were selected. The first sample, called ALG-OPH, contained all patients considered GRAD by these two graders $(n=2496)$. The second sample, called PCP$\mathrm{OPH}$, included all patients considered GRAD by these other pairs of graders $(n=2825)$.

Our data showed that the PCP-OPH group had a mean age of 1.4 years, significantly higher than that of the ALG-OPH group $(p=0.0003)$. No significant differences were detected between the two samples for any of the other variables (Table 6). 
Table 6. Comparison of the study variables ALG-OPH vs. PCP-OPH. NDME = no diabetic macular edema. $\mathrm{DR}=$ diabetic retinopathy. $\mathrm{DME}=$ diabetic macular edema. STDR $=$ sight-threatening DR. * Results with statistically significant differences.

\begin{tabular}{|c|c|c|c|c|c|c|}
\hline \multicolumn{2}{|c|}{ Variable of Interest } & \multicolumn{2}{|c|}{ ALG-OPH $(n=2496)$} & \multicolumn{2}{|c|}{ PCP-ОРН $(n=2825)$} & $p$ Value \\
\hline \multicolumn{2}{|c|}{ Gender (F/M) } & \multicolumn{2}{|c|}{$42.9 \% / 57.1 \%$} & \multicolumn{2}{|c|}{$43.6 \% / 56.4 \%$} & 0.6071 \\
\hline \multicolumn{2}{|c|}{ Age (years) } & \multicolumn{2}{|c|}{$61.4 \pm 14.7$} & \multicolumn{2}{|c|}{$62.8 \pm 14.6$} & 0.0003 * \\
\hline \multicolumn{2}{|c|}{ Years from diagnosis } & \multicolumn{2}{|c|}{$10.2 \pm 7.3$} & \multicolumn{2}{|c|}{$10.3 \pm 7.3$} & 0.6522 \\
\hline \multicolumn{2}{|c|}{ Mydriasis } & \multicolumn{2}{|c|}{$98.3 \%(2454)$} & \multicolumn{2}{|c|}{$98.6 \%(2785)$} & 0.3753 \\
\hline \multirow{3}{*}{$\begin{array}{l}\text { Diabetes } \\
\text { type }\end{array}$} & Type 1 & \multicolumn{2}{|c|}{$14.5 \%(361)$} & \multicolumn{2}{|c|}{$12.8 \%(362)$} & 0.0710 \\
\hline & Type 2 & \multicolumn{2}{|c|}{$85.2 \%(2126)$} & \multicolumn{2}{|c|}{$86.8 \%(2451)$} & 0.0928 \\
\hline & Others & \multicolumn{2}{|c|}{$0.3 \%(9)$} & \multicolumn{2}{|c|}{$0.4 \%(12)$} & 0.5394 \\
\hline \multicolumn{2}{|c|}{ NODR } & \multicolumn{2}{|c|}{$78.6 \%(1961)$} & \multicolumn{2}{|c|}{$79.2 \%(2238)$} & 0.5924 \\
\hline \multicolumn{2}{|c|}{ DR } & \multicolumn{2}{|c|}{$21.43 \%(535)$} & \multicolumn{2}{|c|}{$20.78(587)$} & 0.5619 \\
\hline \multicolumn{2}{|r|}{ Mild } & \multicolumn{2}{|c|}{$10.1 \%(251)$} & \multicolumn{2}{|c|}{$9.5 \%(267)$} & 0.4622 \\
\hline \multirow[t]{3}{*}{ DR stage } & Moderate & $10.9 \%(272)$ & $\begin{array}{c}\text { (NDME } \\
217) \\
(\mathrm{DME} 55)\end{array}$ & $10.8 \%(305)$ & $\begin{array}{c}\text { (NDME } \\
245) \\
(\mathrm{DME} 60)\end{array}$ & 0.9068 \\
\hline & Severe & $0.3 \%(7)$ & $\begin{array}{c}\text { (NDME 1) } \\
\text { (DME 6) }\end{array}$ & $0.3 \%(9)$ & $\begin{array}{c}\text { (NDME 2) } \\
\text { (DME 7) }\end{array}$ & 1 \\
\hline & Proliferative & $0.2 \%(5)$ & $\begin{array}{c}\text { (NDME 2) } \\
\text { (DME 3) }\end{array}$ & $0.2 \%(6)$ & $\begin{array}{c}\text { (NDME 2) } \\
(\mathrm{DME} 4)\end{array}$ & 1 \\
\hline \multirow{2}{*}{\multicolumn{2}{|c|}{$\begin{array}{l}\text { DME } \\
\text { STDR }\end{array}$}} & \multicolumn{2}{|c|}{$2.6 \%(64)$} & \multicolumn{2}{|c|}{$2.5 \%(71)$} & 0.8173 \\
\hline & & \multicolumn{2}{|c|}{$2.7 \%(67)$} & \multicolumn{2}{|c|}{$2.7 \%(75)$} & 1 \\
\hline
\end{tabular}

The frequencies of the matching of the diagnostic categories of each strategy with the criteria of the OPH and the values of indexes (sensitivity, specificity, positive and negative predictive values, likelihood ratios) that determine the validity of the diagnostic tests with the different classifier strategies are shown in Table 7. In particular, the ALG strategy showed a greater ability than the PCP to detect retinopathy in diabetic patients retinographies (Sensitivity, 85.05 vs. 64.54). However, the PCP strategy better identified individuals without retinopathy as demonstrated by its specificity values ( 80.67 vs. 89.59$)$. These results suggest that the diagnostic ability of the ALG was overall superior to that of the PCP.

Table 7. Unpaired sample. Diagnostic category pairs frequency and diagnostic validity indexes.

\begin{tabular}{ccc}
\hline Variable of Interest & ALG-OPH (95\% CI) & PCP-OPH (95\% CI) \\
\hline True Positive & 455 & 373 \\
False Positive & 379 & 233 \\
False Negative & 80 & 214 \\
True Negative & 1582 & 2005 \\
Prevalence & $21.43 \%(19.80-23.06)$ & $20.78 \%(19.26-22.29)$ \\
Sensitivity & $85.05 \%(81.93-88.16)$ & $63.54 \%(59.56-67.52)$ \\
Specificity & $80.67 \%(78.90-82.45)$ & $89.59 \%(88.30-90.88)$ \\
Positive Predictive Value & $54.56 \%(51.12-58.00)$ & $61.55 \% 57.60-65.51)$ \\
Negative Predictive Value & $95.19 \%(94.13-96.25)$ & $90.36 \%(89.11-91.61)$ \\
Likelihood Ratio + & $4.40(3.99-4.85)$ & $6.10(5.33-6.99)$ \\
Likelihood Ratio - & $0.19(0.15-0.23)$ & $0.41(0.37-0.45)$ \\
\hline
\end{tabular}

Four of the 80 DR patients classified as healthy by the ALG were positive for MOD plus DME and in the case of the PCP strategy it was 4 out of 214. The proportion of STDR patients incorrectly diagnosed was 5.9\% (4/67) for the ALG and 5.3\% (4/75) for the PCP. There was no statistically significant difference in the proportions of misdiagnosed 
patients between the two strategies (ALG vs. PCP; $p=0.8693$ ). In fact, the sensitivity of the ALG strategy to diagnose STDR was $94 \%$ and that of PCP 95\%. No patient with severe or proliferative retinopathy was misdiagnosed by ALG or PCP. Both strategies had a low positive predictive value (PPV; Table 7), being even lower for ALG (PPV 54.56 vs. 61.55) but a high negative predictive value (ALG 95.19 vs. PCP 90.36). The ALG strategy not only had a higher sensitivity, but also a better negative predictive value than the PCP strategy.

Likelihood ratios (LR) are another alternative for calculating the diagnostic accuracy and summarizing the information endowed in both sensitivity and specificity. The LR is the probability that a particular test result would be expected among patients with the condition diagnosed compared to the likelihood that that same result would be expected in a patient without the condition. Good diagnostic tests have LR+ $>10$ and their positive result has a significant input to the diagnosis. Good diagnostic tests have LR- $<0,1$. The lower the LR- the more significant contribution of the test is in ruling-out (Table 8)

Table 8. Ranges of likelihood ratio values and their impact on diagnostic accuracy.

\begin{tabular}{ccc}
\hline Likelihood Ratio + & Likelihood Ratio - & Usefulness \\
\hline 10 & $<0.1$ & Highly relevant \\
$5-10$ & $5-10$ & Good \\
$2-5$ & $2-5$ & Fair \\
$<2$ & $<2$ & Poor \\
\hline
\end{tabular}

In the ALG strategy, the modification of the previous probability for positive test results was small $(\mathrm{LH}+4.40$, Table 7$)$. In fact, we calculated the post-test probability using Fagan's nomogram and obtained that the probability of a patient having a DR changed from $21 \%$ to $55 \%$. About 1 in 1.8 positive tests corresponded to patients with retinopathy. The subsequent probability, if the test was negative, was modified to $5 \%$, so approximately 1 of each negative result corresponded to an individual without DR. Likewise, the modification of the previous probability in the PCP test, for positive results, was moderate $(\mathrm{LH}+6.10)$, changing the probability of a patient having a DR from $21 \%$ to $62 \%$ (Fagan's nomogram). About 1 in 1.6 positive tests corresponded to patients with retinopathy. The subsequent probability, if the test is negative, was modified from $21 \%$ to $10 \%$, so approximately 1 in 1.1 negative results corresponded to an individual without retinopathy.

To determine the discriminative power of the different classifier strategies of DR diagnosis, our results were compared using the area under the curve (AUC) of the Receiver Operating Characteristics (ROC) curve (Figure 2).

The AUC for the PCP displayed a value of 0.7657 (95\% CI 0.7452 to 0.7861$)$, while the value of AUC for ALG was 0.8286 (95\% CI 0.8111 to 0.8461$)$. We found that there was a significant difference between the two curves $(p=0.0000)$. Therefore, these power results shown in Figure 2 indicate that the ALG was more efficient than the PCP.

Finally, to assess the intergrader reliability for DR diagnosis, we used the Kappa statistic. We found that the k-value of the agreement between the ALG and OPH was 0.5462 (95\% CI 0.5109 to 0.5815 ), while between PCP and OPH was 0.5251 (95\% CI 0.4865 to 0.5636). The kappa homogeneity test showed a Chi-square value of 0.6252 corresponding to a $p=0.4291$. Therefore, the difference in concordance between the two diagnostic strategies was not statistically significant.

\subsection{Comparison of Diagnostic Tests in Paired Samples}

To confirm the previous results, we studied the patients classified as GRAD by the three diagnostic strategies, removing from the original sample all patients considered UNG for any of the strategies. In Table 9, the proportions and means of the paired sample obtained were described. 


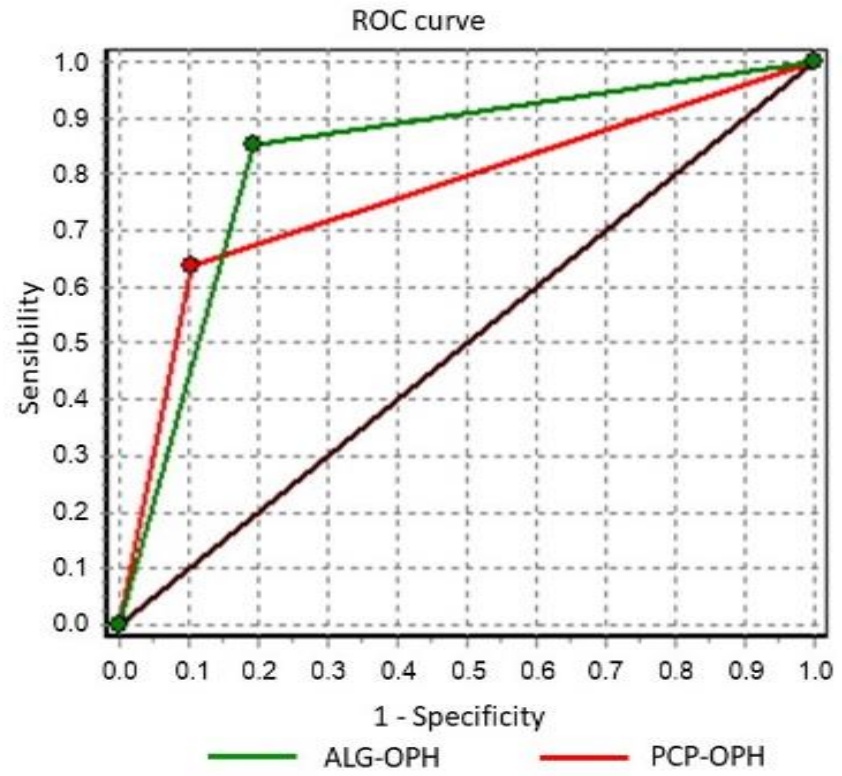

Figure 2. ROC curves comparison. Green line: Algorithm. Red line: Primary Care Physician.

Table 9. Main descriptors of the sample obtained from de APDR for the study between April 2017 and June 2018. ${ }^{*}$ Mean SD. DME = diabetic macular edema. NDME = macular edema non-diabetic. $\mathrm{STDR}=$ sight-threatening DR.

\begin{tabular}{|c|c|c|c|}
\hline \multicolumn{3}{|c|}{ Variable of Interest } & Study Sample $(n=2335)$ \\
\hline \multicolumn{3}{|c|}{ Gender (F/M) } & $42.7 \% / 57.3 \%(997 / 1338)$ \\
\hline \multicolumn{3}{|c|}{ Age (years) } & $61.1 \pm 14.7^{*}$ \\
\hline \multicolumn{3}{|c|}{ Years from diagnosis } & $10.1 \pm 7.2 *$ \\
\hline \multicolumn{3}{|c|}{ Mydriasis (before photograph) } & $98.8 \%(2306)$ \\
\hline \multirow{3}{*}{ Diabetes type } & \multicolumn{2}{|c|}{ Type 2} & $85 \%(1984)$ \\
\hline & \multicolumn{2}{|c|}{ Type 1} & $14.7 \%(343)$ \\
\hline & \multicolumn{2}{|c|}{ Others } & $0.3 \%(8)$ \\
\hline \multicolumn{3}{|c|}{ No Diabetic Retinopathy (NODR) } & $78.9 \%(1842)$ \\
\hline \multicolumn{3}{|c|}{ DR prevalence } & $21.1 \%$ \\
\hline \multirow{7}{*}{$\begin{array}{l}\text { Diabetic retinopathy } \\
\text { (DR) }\end{array}$} & \multicolumn{2}{|c|}{ Mild } & $10.2 \%(238)$ \\
\hline & \multirow{2}{*}{ Moderate } & NDME & $8.3 \%(194)$ \\
\hline & & DME & $2.2 \%(51)$ \\
\hline & \multirow{2}{*}{ Severe } & NDME & $0.04 \%(1)$ \\
\hline & & DME & $0.2 \%(5)$ \\
\hline & \multirow{2}{*}{ Proliferative } & NDME & $0.04 \%(1)$ \\
\hline & & DME & $0.1 \%(3)$ \\
\hline & STDR & & $2.6 \%(61)$ \\
\hline
\end{tabular}

The frequencies of pairs of diagnostic categories of each strategy compared to the criteria of the $\mathrm{OPH}$, and the values of the diagnostic validity indexes for the sample described are displayed in Table 10. 
Table 10. Paired sample. Diagnostic category pairs frequency and diagnostic validity indexes.

\begin{tabular}{ccc}
\hline Variable of Interest & ALG-OPH (95\% CI) & PCP-OPH (95\% CI) \\
\hline True Positive & 418 & 300 \\
False Positive & 351 & 200 \\
False Negative & 75 & 193 \\
True Negative & 1491 & 1642 \\
Prevalence & \multicolumn{2}{c}{$21.1 \%(19.44-22.79)$} \\
Sensibility & $84.8 \%(81.52-88.06)$ & $60.9 \%(56.44-65.26)$ \\
Specificity & $80.9 \%(79.12-82.77)$ & $89.1 \%(87.69-90.59)$ \\
Positive Predictive Value & $54.36 \%(50.77-57.94)$ & $60 \%(55.61-64.39)$ \\
Negative Predictive Value & $95.21 \%(94.12-96.30)$ & $89.48 \%(88.05-90.91)$ \\
Likelihood Ratio + & $4.45(4.02-4.92)$ & $5.60(4.83-6.50)$ \\
Likelihood Ratio - & $0.19(0.15-0.23)$ & $0.44(0.39-0.49)$ \\
\hline
\end{tabular}

The pattern of the indexes shown in Table 10 is comparable to that found in the unpaired sample (Table 7). In fact, the prevalence of DR was the same in all the samples. In summary in the paired sample, the ALG showed a greater ability than PCP to detect DR lesions. However, PCP better identified patients without DR.

Similarly, as in the unpaired sample, we determined that the probability that an individual with a positive test would have retinopathy was small in both strategies (PPV $54.36 \%$ vs. $60 \%$ ), being even lower for the ALG. Both tests presented a high negative predictive value. Therefore, for the unpaired sample, we can conclude that the 2iRetinex software is a good predictor of the absence of DR and a moderate predictor of the presence of DR. In contrast, the diagnostic utility of PCP screening is considered good for positive results and fair for negative.

The AUC for the PCP showed a value of 0.75 , while for the ALG was 0.8287 . We found that there was a significant difference between the two curves (Chi-square value 28.0575, $p=0.0000$ ). Consequently, these results shown in Figure 3 indicate a greater diagnostic power of the ALG.

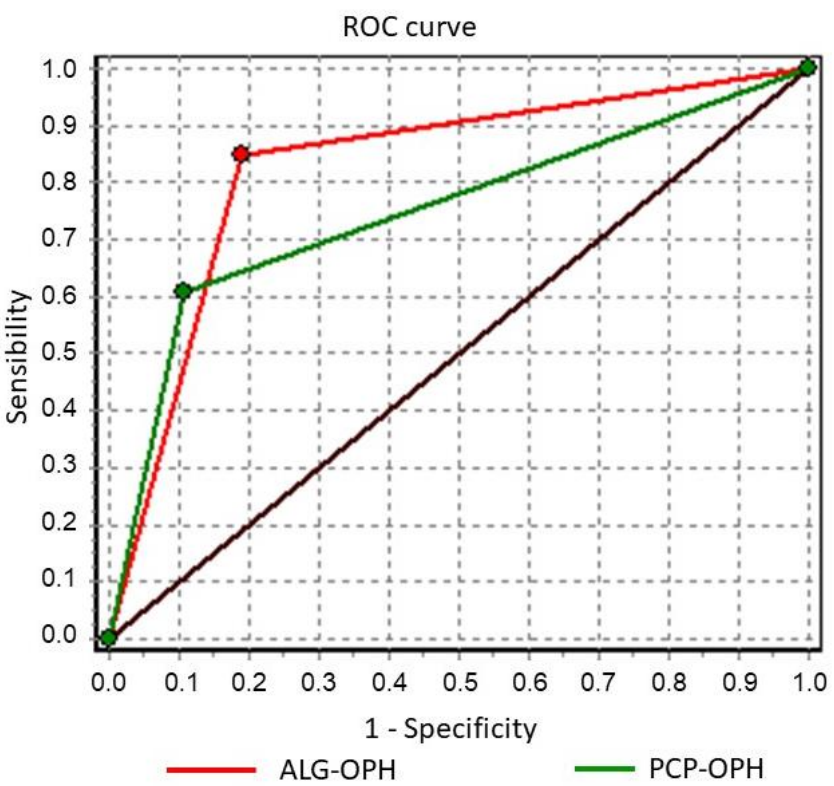

Figure 3. ROC curves comparison of the diagnostic validity. Paired sample. Red: Algorithm. Green: Primary Care Physician.

The k-value of the concordance between the ALG and OPH was 0.5455, while between $\mathrm{PCP}$ and OPH it was 0.4974 . The homogeneity test for the difference in k-values was not significant (Chi-square $2.7898 p=0.949$ ). 


\section{Discussion}

Digital fundus photography is considered an acceptably accurate procedure for detecting DR [13]. Image processing and interpretation in DR screening programs require initial training and ongoing updating for all personnel involved. This entails personal effort and resource consumption. Population-based screening programs for detecting DR help reduce visual loss by identifying sight-threatening cases and referring them to specialists for treatment. A recent meta-analysis of 33 studies worldwide concludes that teleophthalmology has a moderate sensitivity and high specificity for detecting the absence of DR. However, the results obtained for the diagnosis of the diseased retina show widespread variations [19]. In recent years, there has been increasing interest in applying automation processes in ophthalmic telemedicine to reduce the need for screening professionals and to homogenize diagnostic criteria regardless of the origin and composition of the sample being evaluated [20]. For that reason, in this study, we assessed the use of the 2iRetinex software as a complement or substitute for the screening physician.

In this assessment of images from 3520 patients from ten APDR primary care centers, the prevalence of any form of DR was $18.5 \%$, while STDR cases accounted for $2.3 \%$ of the study subjects. Similar results were obtained in the first-year screening of the Scottish program, where the prevalence of DR and STDR was $19.3 \%$ and $1.9 \%$, respectively. These values increased in the subjects reviewed one year (20.5\% and $2.3 \%$, respectively) [21]. Likewise, other studies with similar characteristics showed a percentage of STDR ranging from $2.57 \%$ to $4 \%[8,22,23]$.

According to the American Telemedicine Association Validation Level, the first phase of our APDR screening system can be classified as a category 1 program, with an ondisease/non-disease diagnostic criterion issued by the PCP [24]. Meanwhile, the second phase, characterized by the review of pathological retinographies by the referring ophthalmologist is classified as a category $\mathrm{C} 2$ program. The ophthalmologist establishes the stage and the time frame for the patient's review or for initiating treatment. Previous studies indicated that artifacts may be present in 3-30\% of the photographs without mydriasis [25]. Of note, widespread use of tropicamide ( $97.8 \%$ of patients) does not pose any additional risk [26]. In this regard, the proportion of non-valuable retinal images in published studies is highly dependent on the age distribution of the sample, which in turn is related to the presence of eyelid and corneal abnormalities and especially cataracts. In our study, despite the use of tropicamide to dilate the pupil, the proportion of patients with UNG images was 11 . $6 \%$. Moreover, PCPs were unable to make a judgement on $13.1 \%$ of patients. Likewise, the automatic analysis of the algorithm considered $26.3 \%$ to be unclassifiable. Our data are consistent with those of other studies. In particular, in a validity study, the iGrading automatic assessment system found $26.16 \%$ of 2309 patients to be ungradable [27]. The Italian multicenter study NO BLIND classified $23.4 \%$ of the telediagnostic images as "poor quality non-diagnostic images" [28]. Following this line in another study, the ratio of non-gradable patients classified by the automatic system was twice that of the human classification [22]. Indeed, in this study, 404 patients by the criteria of the automatic system were discarded, and 197 patients by the criteria of the ophthalmologist. Only in two other cases did they agree in classifying them as non-gradable [22]. Similarly, in our study, we found the same lack of diagnostic agreement in image grading between ALG and PCP with respect to OPH ( $\mathrm{k}=0.3623$ and 0.3144 , respectively). This suggests that the mechanisms underlying decision-making are different in algorithms and clinicians. The grading criteria of the automatic systems may have been more demanding than those of the humans, but what is evident is that their implementation is invariable and not affected by the inherent inconsistency of human subjectivity [29].

Our results on the diagnostic accuracy of screening PCP for any grade of DR showed a good mean specificity $(89.6 \%)$ and a reduced sensitivity $(63.5 \%)$. However, for STDR the sensitivity increased to $95 \%$. These values are similar to those reported in other studies $[8,11,26,30]$. Noteworthy, our findings are consistent with a previous partial study about the diagnostic ability of screening PCP at three APDR primary care centers in a small 
sample of patients [17]. Similarly, the concordance in the diagnosis of DR with respect to the clinical judgment of the ophthalmologist was $\mathrm{k}=0.408$ and sensitivity was $97 \%$ and specificity $80 \%$.

The second level of APDR development, which would make it a screening and followup program to monitor patients with mild and moderate retinopathy without macular edema within the program, has not yet been implemented [30]. It could be that the screening physicians want to reduce the workload of ophthalmologists at the second and third levels of care by under-diagnosing cases that do not require referral.

Regarding the results related to the diagnostic accuracy of the 2iRetinex software analysis test (ALG), an overall sensitivity of $85 \%$ was obtained for any degree of retinopathy and a specificity of $81 \%$. For STDR cases, sensitivity increased to $94 \%$. It should be noted that the agreement with the diagnosis of the reference ophthalmologists was similar to that of PCP and the area of the simple ROC curve $(0.8286)$ was slightly similar to that of PCP. Cost-effective ALG screening programs to identify DR lesions on digital images have been reported previously [31]. In particular, Fleming et al. [31] stated that this inclusion of algorithms increased the sensitivity to $100 \%$ in DR detection and reduced manual screening by more than 35\%.

EyeArt, Retmaker, iGrading or IDx are commercially available systems that have been used in teleophthalmic screening programs for DR diagnosis [20]. The functionality of iGrading is comparable to that of $2 \mathrm{iRetinex}$ by combining an image quality system and a DR identification criterion. In the validation study conducted in Valencia [23], iGrading showed excellent sensitivity values of $97.4 \%$ and specificity values of $98.3 \%$ for patients with STDR. For its part, iGrading has been used as a level 1 grading in the Scottish screening program after extensive validation since 2010 with a sensitivity of $97.8 \%$ and specificity of $41.2 \%$ for referable DR [32]. The other two systems, Eyeart and Retmaker showed good diagnostic accuracy with a sensitivity for STDR of $94.7 \%$ and $85 \%$, respectively [33]. These methods qualified as Automated Retinal Image Analysis System (ARIAS) have not yet developed a sufficient level of autonomy to establish a classification of the patient's damage and recommend treatment [34].

ARIAS have been changed with the development of artificial intelligence systems and Deep learning (DL), a subtype of machine learning (ML) that does not require image engineering. Early ML techniques for detecting DR used mathematical image transformation techniques and image engineering [35]. Moreover, DL develops its own pattern recognition representations after being fed raw data [36,37].

In relation to this, it is known that IDx has updated its ARIAS with an artificial intelligence system. The new version IDx-DR v2 is designed to identify DR referable to a specialist without human supervision. IDx-DR v2 achieved $100 \%$ sensitivity and $81.82 \%$ specificity for derivable DR and $100 \%$ sensitivity and $94.64 \%$ specificity for STDR in the sample examined [38]. A meta-analysis demonstrated that ML algorithms have a high diagnostic accuracy for the diagnosis of DR on color fundus photographs suggesting that they may also be ready for clinical application in screening programs [29]. However, early results published in relation to this ML had methodological inconsistencies, such as lack of external validation and the presence of biases. Most of these methods do not provide full interpretations of the relevant findings of retinal pathological signs. Furthermore, it should be noted that, at present, the full implementation of this "black box classification system" presents difficulties for acceptance by clinicians and patients [39].

The main limitation of our study is the dichotomous diagnosis made by the screening PCP. At least at this stage, the APDR screening program is not designed for PCP to classify the DR stage. Indeed, this limitation has prevented us from obtaining full diagnostic validity indexes results in patients with STDR.

The diagnostic capability for STDR stages is excellent for both diagnostic strategies (PCP and ALG). In the case of the overall diagnostic ability for any form of diabetic retinopathy with our algorithm, the $2 \mathrm{iRetinex}$ and PCP strategies are comparable. Moreover, their agreement with respect to the re-evaluation of ophthalmologists does not show 
significant differences. Therefore, the introduction of this real-time software into the APDR workflow would allow knowing whether the images taken of the patient's fundus are of sufficient quality before the patient leaves the examination center. Thus, a re-examination could be recommended if necessary. This strategy would reduce the delay of repeat examinations and consequently, less disrupt the patient's social and work activity. All this suggests that the automatic examination system could be introduced in a real way by integrating it with the screening physician in the first phase.

In this study, we have validated our DR prediction software (2iRetinex) on a sample of patients under real clinical conditions in the routine APDR circuit. Although further assessment is needed to validate the system, it has been confirmed as a tool that could be integrated into DR screening programs. This could improve the quality of screening models in the future. Due to this, studies of the combined use of algorithms and manual classification emerge as an urgent need to achieve better performance. Thereby, the workload of manual classification could be minimized. We plan to conduct studies that focus on extending the algorithm using the 2 iRetinex software to detect other common comorbid eye diseases such as age-related macular degeneration (AMD) and glaucoma.

Author Contributions: S.J.-C. and P.A.-M. were responsible for all aspects of the project including conceptualization and design of the study. S.J.-C., P.A.-M. and P.A.-R. reviewed all the images and stablished the reference diagnosis; E.M. acquired and analyzed APDR data. S.J.-C. and P.A.-M. prepared figures and tables. S.J.-C. and P.A.-M. provided the first draft, prepared final figures, and discussed the results. E.M. and M.A.-D. provided critical review; S.J.-C. and P.A.-M. provided the final version and financial support for the project leading to this publication. All authors have read and agreed to the published version of the manuscript.

Funding: This research was funded by Instituto de Salud Carlos III (FIS PI1601543).

Institutional Review Board Statement: The study was conducted according to the guidelines of the Declaration of Helsinki and approved by the Research and Ethics Committee of Hospital Universitario Puerta del Mar (protocol code PI16-01543, date of approval 26 July 2016).

Informed Consent Statement: Not applicable.

Data Availability Statement: Anonymized data processed in an excel file are available on reasonable request. Restrictions apply to the availability of APDR data. These data are subject to ethical restrictions (Data Protection Act).

Acknowledgments: The authors are grateful for the commitment of the nurses and technicians involved in the appointments and photographs, and of the primary care physicians who contributed with their diagnosis and referrals, to the diabetic retinopathy screening program and this research.

Conflicts of Interest: The authors declare that there is no conflict of interests regarding the publication of this paper.

\section{References}

1. International Diabetes Federation. IDF Diabetes Atlas, 9th ed.; International Diabetes Federation: Brussels, Belgium, 2019.

2. Rodriguez-Acuña, R.; Mayoral, E.; Aguilar-Diosdado, M.; Rave, R.; Oyarzabal, B.; Lama, C.; Carriazo, A.; Asuncion Martinez-Brocca, M. Andalusian program for early detection of diabetic retinopathy: Implementation and 15-year follow-up of a population-based screening program in Andalusia, Southern Spain. BMJ Open Diabetes Res. Care 2020, 8 , 1-8. [CrossRef] [PubMed]

3. Vithian, K.; Hurel, S. Microvascular complications: Pathophysiology and management. Clin. Med. J. R. Coll. Phys. Lond. 2010, 10, 505-509. [CrossRef] [PubMed]

4. Liew, G.; Michaelides, M.; Bunce, C. A comparison of the causes of blindness certifications in England and Wales in working age adults (16-64 years), 1999-2000 with 2009-2010. BMJ Open 2014, 4, 1999-2000. [CrossRef] [PubMed]

5. Yau, J.W.Y.; Rogers, S.; Kawasaki, R.; Lamoreux, E.L.; Kowalski, J.; Bek, T.; Chen, S.-J.; Dekker, J.M.; Fletcher, A.; Grauslund, J.; et al. Global prevalence and major risk factors of diabetic retinopathy. Diabetes Care 2012, 35, 556-564. [CrossRef]

6. Thomas, R.L.; Halim, S.; Gurudas, S.; Sivaprasad, S.; Owens, D.R. IDF Diabetes Atlas: A review of studies utilising retinal photography on the global prevalence of diabetes related retinopathy between 2015 and 2018. Diabetes Res. Clin. Pract. 2019, 157, 107840. [CrossRef] 
7. Lin, K.Y.; Hsih, W.H.; Lin, Y.B.; Wen, C.Y.; Chang, T.J. Update in the epidemiology, risk factors, screening, and treatment of diabetic retinopathy. J. Diabetes Investig. 2021, 12, 1322-1325. [CrossRef]

8. Vujosevic, S.; Aldington, S.J.; Silva, P.; Hernandez, C.; Scanlon, P.; Peto, T.; Simo, R. Screening for diabetic retinopathy: New perspectives and challenges. Lancet Diabetes Endocrinol. 2020, 8, 337-347. [CrossRef]

9. Teo, Z.L.; Tham, Y.C.; Yu, M.; Cheng, C.Y.; Wong, T.Y.; Sabanayagam, C. Do we have enough ophthalmologists to manage vision-threatening diabetic retinopathy? A global perspective. Eye 2020, 34, 1255-1261. [CrossRef]

10. Cunha, L.P.; Figueriedo, E.A.; Pereira Araujo, H.; Ferreira Costa-Cunha, L.V.; Ferreira Costa, C.; de Melo Costa Neto, J.; Mota Freitas Matos, A.; de Oliveira, M.M.; Gomes Bastos, M.; Ribeiro Monteiro, M.L. Non-Mydriatic Fundus Retinography in Screening for Diabetic Retinopathy: Agreement Between Family Physicians, General Ophthalmologists, and a Retinal Specialist. Front. Endocrinol. 2018, 9, 251. [CrossRef]

11. Thapa, R.; Bajimaya, S.; Pradhan, E.; Sharma, S.; Kshetri, B.B.; Paudyal, G. Agreement on grading retinal findings of patients with diabetes using fundus photographs by allied medical personnel when compared to an ophthalmologist at a diabetic retinopathy screening program in nepal. Clin. Ophthalmol. 2020, 14, 2731-2737. [CrossRef]

12. Andonegui, J.; Zurutuza, A.; Pérez de Arcelus, M.; Serrano, L.; Eguzkiza, A.; Auzmendi, M.; Gaminde, I.; Aliseda, D. Diabetic retinopathy screening with non-mydriatic retinography by general practitioners: 2-Year results. Prim. Care Diabetes 2012, 6 , 201-205. [CrossRef] [PubMed]

13. Begum, T.; Rahman, A.; Nomani, D.; Mamun, A.; Adams, A.; Islam, S.; Khair, Z.; Khair, Z.; Answar, I. Diagnostic accuracy of detecting diabetic retinopathy by using digital fundus photographs in the peripheral health facilities of Bangladesh: Validation study. JMIR Public Health Surveill. 2021, 7, 1-14. [CrossRef] [PubMed]

14. Katada, Y.; Ozawa, N.; Masayoshi, K.; Ofuji, Y.; Tsubota, K.; Kurihara, T. Automatic screening for diabetic retinopathy in interracial fundus images using artificial intelligence. Intell. Med. 2020, 3-4, 100024. [CrossRef]

15. Rogers, T.W.; Gonzalez-Bueno, J.; Garcia Franco, R.; Lopez Star, E.; Mendez Marin, D.; Vassallo, J.; Lansingh, V.C.; Trikha, S.; Jaccard, N. Evaluation of an AI system for the detection of diabetic retinopathy from images captured with a handheld portable fundus camera: The MAILOR AI study. Eye 2021, 35, 632-638. [CrossRef]

16. Mataix, B.; Ponte, B. Teleophthalmology as screening method for diabetic retinopathy. Av. Diabetol. 2009, 25, $209-212$.

17. Vargas-Sánchez, C.; Maldonado-Valenzuela, J.J.; Pérez-Durillo, F.T.; González-Calvo, J.; Pérez-Milena, A. Cribado de retinopatía diabética mediante retinografía midriática en atención primaria. Salud Pública México 2011, 53, 212-219.

18. Wilkinson, C.P.; Ferris, F.L., III; Klein, R.E.; Lee, R.P.; Agardh, C.D.; Davis, M.; Dills, D.; Kampik, A.; Pararajasegaram, A.; Verdaguer, J.T.; et al. Proposed international clinical diabetic retinopathy and diabetic macular edema disease severity scales. Ophthalmology 2003, 110, 1677-1682. [CrossRef]

19. Ullah, W.; Pathan, S.H.; Panchal, A.; Anandan, S.; Saleem, K.; Sattar, Y.; Ahmad, E.; Mukhtar, M.; Nawaz, H. Cost-effectiveness and diagnostic accuracy of telemedicine in macular disease and diabetic retinopathy: A systematic review and meta-analysis. Medicine 2020, 99, e20306. [CrossRef]

20. Sim, D.A.; Keane, P.A.; Tufail, A.; Egan, C.A.; Aiello, L.P.; Silva, P.S. Automated Retinal Image Analysis for Diabetic Retinopathy in Telemedicine. Curr. Diabetes Rep. 2015, 15, 14. [CrossRef]

21. Looker, H.C.; Nyangoma, S.O.; Cromie, D.; Olson, J.A.; Leese, G.P.; Black, M.; Doig, J.; Lee, N.; Lindsay, R.S.; McKnight, J.A.; et al. Diabetic retinopathy at diagnosis of type 2 diabetes in Scotland. Diabetologia 2012, 55, 2335-2342. [CrossRef]

22. Shah, A.; Clarida, W.; Amelon, R.; Hernaez-Ortega, M.C.; Navea, A.; Morales-Olivas, J.; Dolz-Marco, R.; Verbaak, F.; Jorda, P.P.; van der Heijden, A.A.; et al. Validation of Automated Screening for Referable Diabetic Retinopathy with an Autonomous Diagnostic Artificial Intelligence System in a Spanish Population. J. Diabetes Sci. Technol. 2020, 15, 655-663. [CrossRef] [PubMed]

23. Soto-Pedre, E.; Navea, A.; Millan, S.; Hernaez-Ortega, M.C.; Morales, J.; Desco, M.C.; Perez, P. Evaluation of automated image analysis software for the detection of diabetic retinopathy to reduce the ophthalmologists' workload. Acta Ophthalmol. 2015, 93, e52-e56. [CrossRef] [PubMed]

24. Tozer, K.; Woodward, M.A.; Newman-Casey, P.A. Telemedicine and Diabetic Retinopathy: Review of Published Screening Programs. J. Endocrinol. Diabetes 2015, 2. [CrossRef]

25. Scanlon, H.; Stephen, J. The influence of age, duration of diabetes, cataract, and pupil size on image quality in digital photographic retinal screening. Diabetes Care 2005, 28, 2448-2453. [CrossRef] [PubMed]

26. Pandit, R.J.; Taylor, R. Mydriasis and glaucoma: Exploding the myth. A systematic review. Diabetes Med. 2000, 17, 693-699. [CrossRef] [PubMed]

27. Scarpa, G.; Urban, F.; Vujosevic, S.; Tessarin, M.; Gallo, G.; Vissentin, A.; Foglia, E.; Ferrario, L.; Midena, E. The Nonmydriatic Fundus Camera in Diabetic Retinopathy Screening: A Cost-Effective Study with Evaluation for Future Large-Scale Application. J. Ophthalmol. 2016, 2016, 4625096. [CrossRef]

28. Sasso, F.C.; Pafundi, P.C.; Gelso, A.; Bono, V.; Costagliola, C.; Marfella, R.; Sardu, C.; Rinaldi, L.; Galiero, R.; Acierno, C.; et al. Telemedicine for screening diabetic retinopathy: The NO BLIND Italian multicenter study. Diabetes Metab. Res. Rev. 2019, 35, e3113.

29. Wu, J.-H.; Liu, T.-Y.A.; Hsu, W.-T.; Ho, J.H.-C.; Lee, C.-C. Performance and limitation of machine learning algorithms for diabetic retinopathy screening: A meta-analysis. J. Med. Internet Res. 2020, 23, e23863. [CrossRef]

30. Oquendo, M.V.I. Programa de Detección Precoz de la Retinopatía Diabética en Andalucía. Investig. Diabetes 2013, 33-37. Available online: http:/ / www.diabetespractica.com/files/docs/publicaciones/138512840910_Iborra.pdf (accessed on 23 October 2021). 
31. Fleming, A.D.; Goatman, K.A.; Phillip, S.; Williams, G.J.; Prescott, G.J.; Scotland, G.S.; McNamee, P.; Leese, G.; Wykes, W.; Sharp, P.F.; et al. The role of haemorrhage and exudate detection in automated grading of diabetic retinopathy. Br. J. Ophthalmol. 2010, 94, 706-711. [CrossRef]

32. Philip, S.; Fleming, A.D.; Goatman, K.A.; Fonseca, S.; McNamee, P.; Scotland, G.S.; Prescott, G.J.; Sharp, P.F.; Olson, J.A. The efficacy of automated 'disease/no disease' grading for diabetic retinopathy in a systematic screening programme. Br. J. Ophthalmol. 2007, 91, 1512-1517. [CrossRef] [PubMed]

33. Tufail, A.; Rudisill, C.; Egan, C.; Kapetanakis, V.V.; Salas-Vega, S.; Owen, C.G.; Lee, A.; Louw, V.; Anderson, J.; Liew, G.; et al Automated Diabetic Retinopathy Image Assessment Software: Diagnostic Accuracy and Cost-Effectiveness Compared with Human Graders. Ophthalmology 2017, 124, 343-351. [CrossRef]

34. Abràmoff, M.D.; Leng, D.; Ting, D.S.W.; Rhee, K.; Horton, M.B.; Brady, C.J.; Chiang, M.F. Automated and Computer-Assisted Detection, Classification, and Diagnosis of Diabetic Retinopathy. Telemed. eHealth 2020, 26, 544-550. [CrossRef]

35. Wernick, M.N.; Yang, Y.; Brankov, J.G.; Yourganov, G.; Strother, S.C. Drawing conclusions from medical images. IEEE Signal Process. Mag. 2010, 25, 25-38. [CrossRef]

36. Abbas, Q.; Fondon, I.; Sarmiento, A.; Jiménez, S.; Alemany, P. Automatic recognition of severity level for diagnosis of diabetic retinopathy using deep visual features. Med. Biol. Eng. Comput. 2017, 55, 1959-1974. [CrossRef] [PubMed]

37. Esteva, A.; Robicquet, A.; Ramsundar, B.; Kuleshov, V.; DePristo, M.; Chou, K.; Cui, C.; Corrado, G.; Thrun, S.; Dean, J. A guide to deep learning in healthcare. Nat. Med. 2019, 25, 24-29. [CrossRef] [PubMed]

38. Peris-Martínez, C.; Shaha, A.; Clarida, W.; Amelon, R.; Hernáez-Ortega, M.C.; Navea, A.; Morales-Olivas, J.; Dolz-Marco, R.; Pérez-Jordá, P.; Verbraak, F.; et al. Use in clinical practice of an automated screening method of diabetic retinopathy that can be derived using a diagnostic artificial intelligence system. Arch. Soc. Esp. Oftalmol. 2021, 96, 117-126. [CrossRef]

39. Colomer, A.; Igual, J.; Naranjo, V. Detection of early signs of diabetic retinopathy based on textural and morphological information in fundus images. Sensors 2020, 20, 1005. [CrossRef] [PubMed] 